\title{
Desempeño y calidad de servicio de autobuses externos de la Universidad de Costa Rica
}

\section{Transit performance and quality of service indicators for transit services for students to the Universidad de Costa Rica}

\section{Gabriel Chaves-Flores}

Escuela de Ingeniería Civil, Universidad de Costa Rica

gabriel.chavesflores@ucr.ac.cr

\section{Henry Hernández-Vega}

Laboratorio Nacional de Materiales y Modelos Estructurales, LanammeUCR, Universidad de Costa Rica

henry.hernandezvega@ucr.ac.cr

Fecha de recepción: 27 de octubre de 2015 / Fecha de aprobación: 23 de noviembre de 2015

\section{RESUMEN}

Se presenta la aplicación de una metodología para la evaluación de un sistema de transporte público, modalidad autobús. En la evaluación se aplican tanto indicadores de desempeño como indicadores relacionados con la calidad del servicio para analizar la movilidad de estudiantes y del personal de la sede Rodrigo Facio de la Universidad de Costa Rica (UCR) que utilizan el servicio especial de autobuses de la universidad conocido como buses externos UCR. Más de una tercera parte de los viajes al centro académico se realizan por medio del servicio especial de autobuses; lo que implica que la calidad de la movilidad de más de 10 mil personas por día depende de este servicio. La información del desempeño y calidad de servicio del sistema de transporte público es elemental para futuros planes maestros de movilidad en la universidad. En un día promedio entre semana se realizan más de 350 viajes en autobús (bus externo UCR), incluyendo viajes de salida y llegada a la Sede Rodrigo Facio por lo que se debe ofrecer un transporte público capaz de atraer nuevos usuarios y así contribuir a generar un ambiente agradable y saludable. Se lograron identificar los aspectos que más aquejan al usuario. La aplicación de la metodología permitió evaluar el sistema a través de diferentes indicadores de desempeño a partir de información disponible. Finalmente, dentro de los principales hallazgos se encuentran posibles nuevas rutas y una lista de medidas para mejorar la calidad del servicio, de acuerdo a la importancia dada por los usuarios.

PALABRAS CLAVES: Transporte público, perspectiva del usuario, calidad de servicio, indicadores, desempeño.

\section{ABSTRACT}

This paper presents a methodology to evaluate an university bus transit service. Both performance and quality of service indicators were applied in the system evaluation to analyze the mobility of students and staff at the main campus Rodrigo Facio of the University of Costa Rica (UCR), who daily commute using a special bus transit service. Over one third of the total trips to the campus are made by this special bus transit service provided by the university; which implies that the quality of the mobility of more than 10 thousand people depends on this service. Information related to the performance and quality of service of the bus transit system is a fundamental input for future university transportation master plans. On an average weekday more than 350 trips are made by the exclusive bus transit service, including trips that depart and arrive to the main campus Rodrigo Facio; therefore the need to offer a high-quality service in order to increase ridership, and thus, to help to generate a more pleasant and healthier environment. Relevant issues that affect users were identified. Also, the methodology was applied to assess the system through different performance indicators. Finally, the main findings include potential new bus transit routes and a list of countermeasures, ranked according to the priority given by the users to improve the quality of the service.

KEYWORDS: Transit, user perspective, quality of service, indicators, performance. 


\section{INTRODUCCIÓN}

Los aumentos de la congestión del tráfico resultan en tiempos de viaje más largos provocando una pérdida de confiabilidad, además de mayores tarifas, en el transporte público. El transporte público presenta menores emisiones por pasajero-kilómetro transportado comparado con otros medios de transporte (Schipper, Saenger, y Sudardshan, 2011). Además, Ortúzar (2000) indica que el autobús es más eficiente que el vehículo privado en la utilización del espacio vial (pág. 17).

La recopilación de mediciones es fundamental para la adecuada administración de un sistema de transporte. Diferentes medidas pueden brindar diversas perspectivas del desempeño de un sistema de transporte y no existe un único método o indicador que comunique de manera global toda la información para la toma de decisiones (Litman, 2003).

Adicionalmente, Eboli y Mazzulla (2011) establecen que considerar la percepción de las personas que utilizan el autobús como modo de transporte es relevante para evaluar el desempeño de un servicio de transporte público. Aunado a lo anterior, una buena calidad de servicio puede ayudar a retener pasajeros de autobús que tienen otras opciones de transporte (TCRP, 2013).

Por otro lado, Mohring (1972) establece que el costo total de proveer el servicio, incluye cuatro componentes: los costos operativos de la compañía de autobuses y los costos (tiempos) que asume el pasajero al caminar para llegar a la parada, para esperar al bus y el tiempo viajando dentro del autobús. Tal como lo establece Van Reeven (2008) la suma de los costos de espera y tarifa deben ser suficientemente bajos para inducir a los usuarios para que tomen el autobús.

Muchas ciudades en Latinoamérica, y a nivel global, han priorizado el transporte público dentro de las políticas de movilidad y ciudad sostenible (Pardo, 2009). La movilidad urbana se ha convertido en una de los principales preocupaciones sociales (Iracheta, 2011). Costa Rica requiere un mejoramiento constante del transporte dado el impacto que este tiene en la calidad de vida y en la eficiencia de los viajes. INECO (2011) estima que en el país se realizan más de dos millones de viajes diarios en transporte público de pasajeros. El ineficiente sistema de transporte público existente combinado con un aumento del parque automotor se encuentran entre las principales causas que generan pérdidas económicas en la Gran Área Metropolitana (Otoya, 2013). Además, de acuerdo con Brenes y Rodríguez (2008) se requiere una modernización y reordenamiento del transporte público en la Gran Área Metropolitana.
De acuerdo con Leandro, Molina y Riba (2013) el incremento de la flota vehicular en el país también ha impactado a la sede Rodrigo Facio de la Universidad de Costa Rica (UCR). Indican además, que el mejoramiento del servicio de transporte público universitario es un aspecto a intervenir dentro de una política de universidad saludable. Para lograr mejoras en este servicio es necesario tener una línea de base que permita establecer las condiciones actuales de operación. Por esto, nos planteamos la siguiente pregunta: ¿cuál es el desempeño y la percepción del servicio de transporte de estudiantes, en autobús, de la Sede Rodrigo Facio de la UCR en el año 2015?

\section{OBJETIVO GENERAL}

Aplicar indicadores de desempeño y calidad del servicio de sistema de transporte de estudiantes de la Sede Rodrigo Facio de la Universidad de Costa Rica con el fin de forjar herramientas que permitan evaluar y mejorar la planificación del sistema de transporte público especial modalidad autobús.

\section{METODOLOGIA}

Se inició con una revisión de la literatura y se consideraron disposiciones de la Autoridad Reguladora de Servicios Públicos (ARESEP) y del Consejo de Transporte Público (CTP). En la fase inicial se debió comenzar a filtrar la información y establecer el alcance del proyecto. Durante el desarrollo del proyecto se determinaron parámetros como horas de servicio, frecuencia, cantidad de viajes, costo por kilómetro, demanda insatisfecha; además se aplicó una encuesta a 380 personas, esta fue realizada con ayuda de distintos estudiantes de la universidad entre los meses de mayo y junio del 2015.

De acuerdo con Contreras (1982), el método de "sube y baja" permite visualizar el funcionamiento de una ruta de autobuses. Para el análisis de demanda se utilizó este método, en tres rutas seleccionadas, una vez por cada sentido (destino-UCR/UCRdestino). Se contabilizaron las personas que subieron y bajaron en cada parada, así como el tiempo asociado a esto. Además, se identificaron lugares donde no se brindan las condiciones necesarias que requiere para viajar con seguridad y confort.

Para finalizar, la información recopilada permite detectar las potenciales intervenciones requeridas para mejorar el servicio de trasporte. De igual manera con este proyecto se pretende fijar antecedentes en las políticas de trasportes en la Gran Área Metropolitana para la aplicación de parámetros que podrían hacer más atractivo el uso del sistema para los usuarios. 


\section{CALIDAD DEL TRANSPORTE PÚBLICO}

La calidad de servicio del transporte público es definida de acuerdo con TCRP (2003) como "el desempeño general del servicio de transporte púbico desde el punto de vista del usuario" (página 4-6). La calidad del servicio refleja la satisfacción de las necesidades de los individuos. Los indicadores son seleccionados en función de la importancia para el usuario, la factibilidad de ser calculados y que los datos necesarios para su obtención estén disponibles tanto en el presente como en el futuro. Entre ellos se encuentran: tiempo de viaje, velocidad de viaje de los usuarios, razón de distancia en ruta, etapas por viaje, velocidad bus versus vehículo privado y transbordos críticos.

La calidad del servicio refleja que tan bien el servicio de transporte público satisface las necesidades de los usuarios. Sin embargo, un balance es necesario entre los deseos de los usuarios y el servicio que se puede proveer de una manera razonable o factible en términos económicos (TCRP, 2013).

De acuerdo con el Reporte 88 del TCRP (2002), el transporte público, como una industria de servicio que es, debe entender y trabajar por mejorar las necesidades de los usuarios, incluyendo tanto pasajeros actuales, como los potenciales e involucrar a la comunidad en su proceso de planificación. Además, indica que la evaluación del desempeño debe incluir diferentes perspectivas tales como: usuario, proveedor del servicio, comunidad e interacción autobús y vehículos privados. Iracheta (2011) establece que modernizar al transporte público requiere la incorporación de todos los actores sociales implicados.

Desde la perspectiva del usuario son de interés los siguientes aspectos:

- $\quad$ Seguridad durante el viaje: Registro de accidentes, accidentes de usuarios, vandalismo, delincuencia y dispositivos de seguridad.

- Prestación de servicio: Confiabilidad de horarios, confort durante el viaje y satisfacción del usuario.

- Disponibilidad: Cobertura, frecuencias, horas de servicio y viajes no efectuados.

- Tiempo de viaje: Tiempo de transbordo y tiempo de viaje.

El proveedor del servicio se preocupa porque el servicio se brinde de manera eficiente y efectiva, haciendo énfasis en:

- Impacto al tránsito: Consecuencias en la comunidad, empleo, consecuencias el medio ambiente y movilidad urbana.
- Economía: Número de pasajeros, mantenimiento de la flota, eficiencia de los costos y rentabilidad.

- Mantenimiento y estado de la unidad: Limpieza de la flota, unidades de refuerzo y capacidad de comunicación en carretera.

Aspectos que son de interés de la comunidad:

- Reducción de la congestión y contaminación ambiental.

- Accesibilidad a fuentes de trabajo.

- Posibilidades de viaje cuando el vehículo privado no es opción.

- Provisión de transporte para personas (por ejemplo, adultos mayores y estudiantes) que no tienen acceso al transporte privado.

Interacción autobús y vehículos privados. Dado que la mayoría de los viajes se realizan en corredores de tráfico mixto, es necesario cuantificar el efecto que tienen los autobuses en los flujos de tráfico y viceversa:

- Tiempo de viaje: Retrasos y velocidad de operación.

- Relación volumen/capacidad de carreteras.

TCRP (2002) también indica que tener cuidado al utilizar medidas usualmente utilizadas por ingenieros de tráfico. Por ejemplo, para establecer los tiempos de los semáforos usualmente se toman en cuenta las demoras por vehículo; sin embargo, no se toma en cuenta que la demora total es mayor para los autobuses, asumiendo que en promedio los ocupantes del autobús son muchos más que los de los vehículos privados.

\section{INDICADORES PARA MEDIR LA CALIDAD $Y$ EFICIENCIA EN EL TRANSPORTE PÚBLICO}

En esta investigación se emplean indicadores de desempeño, que son medidas de acuerdo a los parámetros que establece TCRP (2002) en la guía para el desarrollo de un sistema de indicadores de desempeño para el transporte público incluyendo una encuesta para conocer la percepción del individuo referente al servicio prestado.

Es necesario generar mecanismos de transporte público capaces de promover Gestión de Demanda para los usuarios, es ahí donde el término de eficiencia del transporte público se vuelve trascendental. La condición del servicio del transporte público es de esperar que sea de "alta calidad, eficiente, poco contaminante, seguro, cómodo y accesible (Burgos, Silva, Troncoso y Franco, 2013). 
Ver Tabla 1, la cual muestra los principales indicadores de desempeño que se muestran en el TCRP (2002), algunos de ellos se explican brevemente.

Tabla 1. Indicadores de desempeño

\begin{tabular}{|c|c|}
\hline \multicolumn{2}{|c|}{ Principales indicadores de desempeño } \\
\hline Distancia entre rutas & $\begin{array}{l}\text { Rendimiento puntual para rutas } \\
\text { fijas }\end{array}$ \\
\hline Cobertura & Regularidad de avance \\
\hline Cobertura de rutas & Viajes cancelados \\
\hline Densidad del servicio & Viajes perdidos \\
\hline $\begin{array}{l}\text { Porcentaje de personas por } \\
\text { minuto que utilizan el Servicio }\end{array}$ & $\begin{array}{l}\text { Porcentaje de rutas programadas } \\
\text { que están prestando el servicio }\end{array}$ \\
\hline Accesibilidad en las paradas & $\begin{array}{l}\text { Kilómetros programados por } \\
\text { minuto de atraso }\end{array}$ \\
\hline Frecuencia & Equipamiento del autobús \\
\hline Lapso del servicio & Antigüedad de la flota \\
\hline Horas de servicio & $\begin{array}{l}\text { Presencia de quejas o } \\
\text { comentarios positivos }\end{array}$ \\
\hline $\begin{array}{l}\text { Horas en que se perciben } \\
\text { ingresos }\end{array}$ & $\begin{array}{l}\text { Tiempo de respuesta del servicio } \\
\text { al cliente }\end{array}$ \\
\hline Tiempo de respuesta & Amabilidad del conductor \\
\hline Composición de la flota & Confort del pasajero \\
\hline Porcentaje de rutas programadas & $\begin{array}{c}\text { Porcentaje de paradas con techo } \\
\text { y bancas }\end{array}$ \\
\hline Distancia entre paradas & Satisfacción del usuario \\
\hline $\begin{array}{l}\text { Número de medios de venta de } \\
\text { tiquetes }\end{array}$ & Pasajeros a bordo \\
\hline Servicios denegados & Generación de viajes \\
\hline Personas sin abordar & Análisis demográfico \\
\hline Capacidad en asientos & Accesibilidad \\
\hline
\end{tabular}

Fuente: Basado en TCRP, 2002. Modificado por los autores

\section{Frecuencia}

Corresponde al número de unidades que brindan el servicio por hora o por día. Es necesario obtener la cantidad de autobuses por día, así como el intervalo en que operan. Ver Tabla 2 y Tabla 3.

Tabla 2. Niveles de servicio de acuerdo a los intervalos entre cada unidad para viajes en zonas de alta densidad

\begin{tabular}{|c|c|}
\hline Nivel de servicio & Condición \\
\hline A & Intervalos menores a 10 minutos \\
\hline B & Intervalos entre 10-14 minutos \\
\hline C & Intervalos entre 15-20 minutos \\
\hline D & Intervalos entre 21-30 minutos \\
\hline E & Intervalos entre 31-60 minutos \\
\hline F & Intervalos mayores a 60 minutos \\
\hline
\end{tabular}

Fuente: TCRP, 2003.
Tabla 3. Niveles de servicio para viajes entre ciudades o servicios especiales se puede utilizar este rango de niveles de servicio

\begin{tabular}{|c|c|}
\hline Nivel de servicio & Cantidad de viajes por día \\
\hline A & Más de 15 \\
\hline B & $12-15$ \\
\hline C & $8-11$ \\
\hline D & $4-7$ \\
\hline E & $2-3$ \\
\hline F & $0-1$ \\
\hline
\end{tabular}

Fuente: TCRP, 2003.

\section{Horas de servicio}

Está relacionado con cuanto tiempo al día se provee el servicio. Las horas de servicio pueden variar de acuerdo al día de la semana, la ruta e incluso según las paradas. Existen rutas que pueden brindar sólo el servicio durante las horas pico, pues los flujos provocan que sólo a esas horas el servicio sea rentable. Ver Tabla 4.

Tabla 4. Niveles de servicio de acuerdo a las horas de servicio

\begin{tabular}{|c|c|}
\hline Nivel de servicio & Condición \\
\hline A & $19-24$ horas por día \\
\hline B & $17-18$ horas por día \\
\hline C & $14-16$ horas por día \\
\hline D & $12-13$ horas por día \\
\hline E & $4-11$ horas por día \\
\hline F & $0-3$ horas por día \\
\hline
\end{tabular}

Fuente: TCRP, 2003.

\section{Pasajeros a bordo}

Corresponde al número de pasajeros que están a bordo del autobús, éste indicador es de lo más significativos a la hora de considerar el confort en el viaje, pues la comodidad del usuario está relacionada con el campo que tenga disponible para su viaje. Ver Tabla 5.

Tabla 5. Niveles de servicio asociados al estado en que viajan los pasajeros

\begin{tabular}{|c|c|}
\hline Nivel de servicio & Condición \\
\hline A & Pasajeros no necesitan ir con alguien al lado \\
\hline B & $\begin{array}{l}\text { Pasajeros pueden escoger con quién sentarse a la } \\
\text { par }\end{array}$ \\
\hline C & Todos los pasajeros pueden sentarse \\
\hline D & Algunos pasajeros deben ir de pie \\
\hline $\mathrm{E}$ & $\begin{array}{l}\text { Capacidad máxima del bus, tanto de pie como } \\
\text { sentados }\end{array}$ \\
\hline $\mathrm{F}$ & Sobrepasa capacidad establecida \\
\hline
\end{tabular}

Fuente: TCRP, 2003. 
UN CASO DE APLICACIÓN. SERVICIO ESPECIAL DE TRANSPORTE DE ESTUDIANTES EN LA SEDE RODRIGO FACIO DE LA UNIVERSIDAD DE COSTA RICA

La Sede Rodrigo Facio alberga, más de 31 mil estudiantes activos, cuya distribución de provincia de origen se muestra en la Tabla 6. Más de una tercera parte (34\%) de los viajes a la Sede Rodrigo Facio de la Universidad de Costa Rica se hacen a través del sistema de servicio especial de transporte de estudiantes de la universidad (Hernández y Jiménez, 2013) conocido como bus externo UCR.

Tabla 6. Distribución población estudiantil en la sede Rodrigo Facio de acuerdo a la provincia de procedencia

\begin{tabular}{|c|c|}
\hline Provincia & $\begin{array}{c}\text { Porcentaje de la población estudiantil en la } \\
\text { sede Rodrigo Facio }\end{array}$ \\
\hline San José & $54 \%$ \\
\hline Alajuela & $14 \%$ \\
\hline Cartago & $14 \%$ \\
\hline Heredia & $12 \%$ \\
\hline Otras & $6 \%$ \\
\hline
\end{tabular}

Fuente: Información suministrada por la Oficina de Registro e Información, 2015. Adecuada por los autores, 2015.

El servicio especial de transporte de estudiantes (bus externo UCR), genera al menos 1840 viajes de autobuses a la semana, incluyendo viajes de entrada y salida al campus. Para determinar las condiciones en que operan las rutas de autobuses se aplican una serie de indicadores de desempeño, y una encuesta de percepción de calidad, con el objetivo de evaluar el sistema actual.

La Figura 1, muestra la repartición modal considerando únicamente los tres principales medios de transporte motorizado hacia la ciudad universitaria.
La provincia de San José cuenta con la mayor proporción de usuarios que viajan en vehículo privado. Por otro lado, la provincia de Cartago cuenta con la mayor proporción de viajes en transporte público regular que podría deberse a la cercanía de las paradas de buses regulares existentes en las cercanías del campus. La provincia de Heredia cuenta con la mayor proporción de viajes en Autobús UCR, a pesar de que solamente existe una ruta disponible.

La Figura 2 muestra la distribución de viajes en autobús y vehículo privado en los cantones de Heredia. Los datos desagregados muestran que es viable analizar la posibilidad de separar la ruta de Heredia, al menos para los periodos pico, haciendo una ruta que podría pasar por los cantones de San Rafael, San Pablo y Santo Domingo, dado que la proporción de usuarios para estos tres cantones iguala a la proporción de usuarios del cantón de Heredia.

Para el segundo semestre del 2015, de acuerdo a la información disponible se encontraron diferencias en los costos para el pasajero por kilómetro. Por ejemplo rutas como San Carlos y San Ramón tienen tarifas similares y comparten un mismo corredor pero tienen una diferencia en el recorrido de más de 30 kilómetros.

\section{Análisis de Demanda Mediante Método Sube y Baja}

El método sube y baja permite determinar la cantidad de pasajeros en un autobús. En la Figura 3 se observa un conteo de pasajeros durante un recorrido en la ruta Heredia-UCR, el autobús alcanza su capacidad de asientos en la primera parada, haciéndolo poco atractivo a usuarios en paradas posteriores. Los análisis de sube y baja permiten identificar, en conjunto con estudios de velocidad

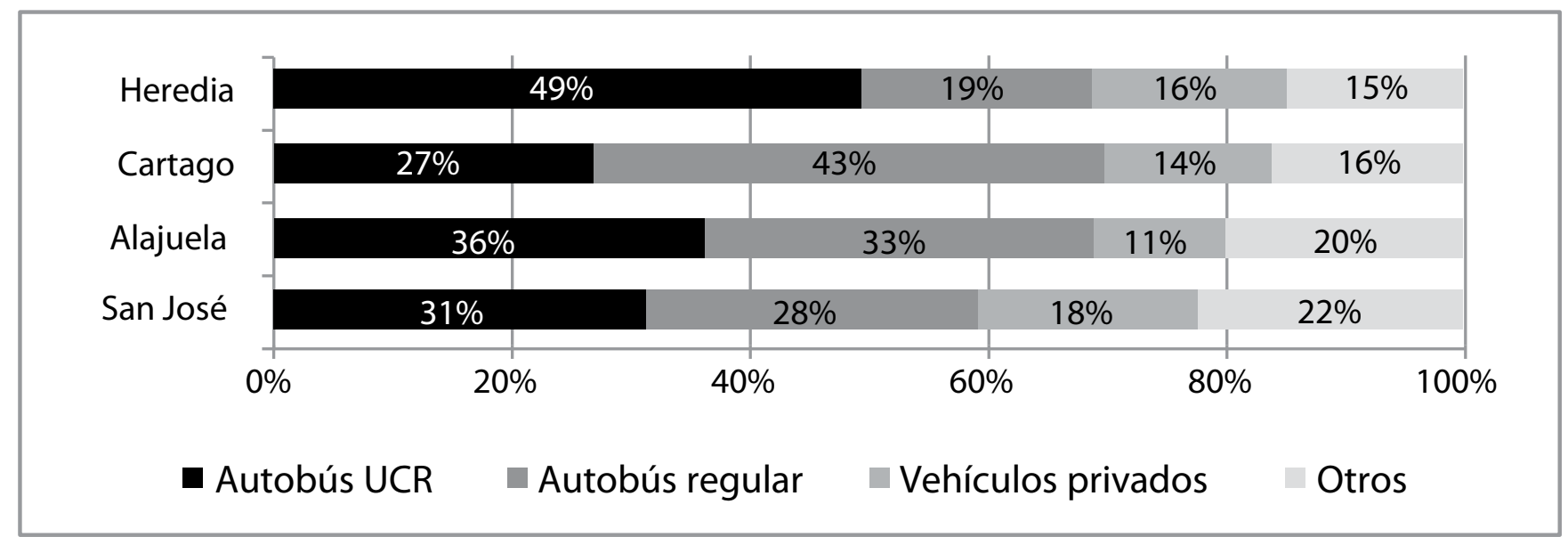

Figura 1. Distribución de viajes hacia el campus Rodrigo Facio por medio de transporte por provincia.

'Entiéndase por "Autobús UCR" como las rutas de servicio especial de transporte de estudiantes que se brinda en la sede Rodrigo Facio de la Universidad de Costa Rica (bus externo UCR). Otros incluye viajes caminando, en tren, en bicicleta, en taxi o medio no especificado. 


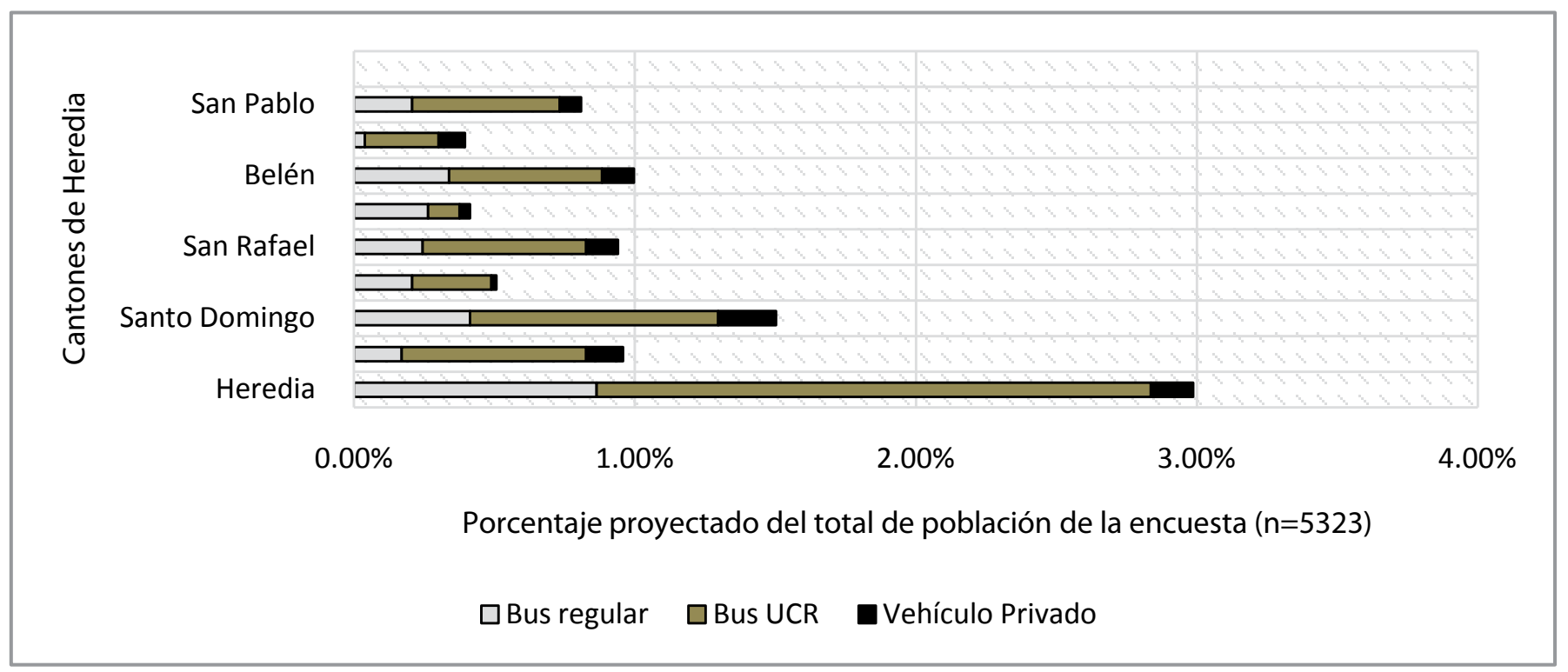

Figura 2. Distribución de medio de transporte en los cantones de la provincia de Heredia. Datos suministrados por el Programa de Infraestructura del Transporte, LanammeUCR

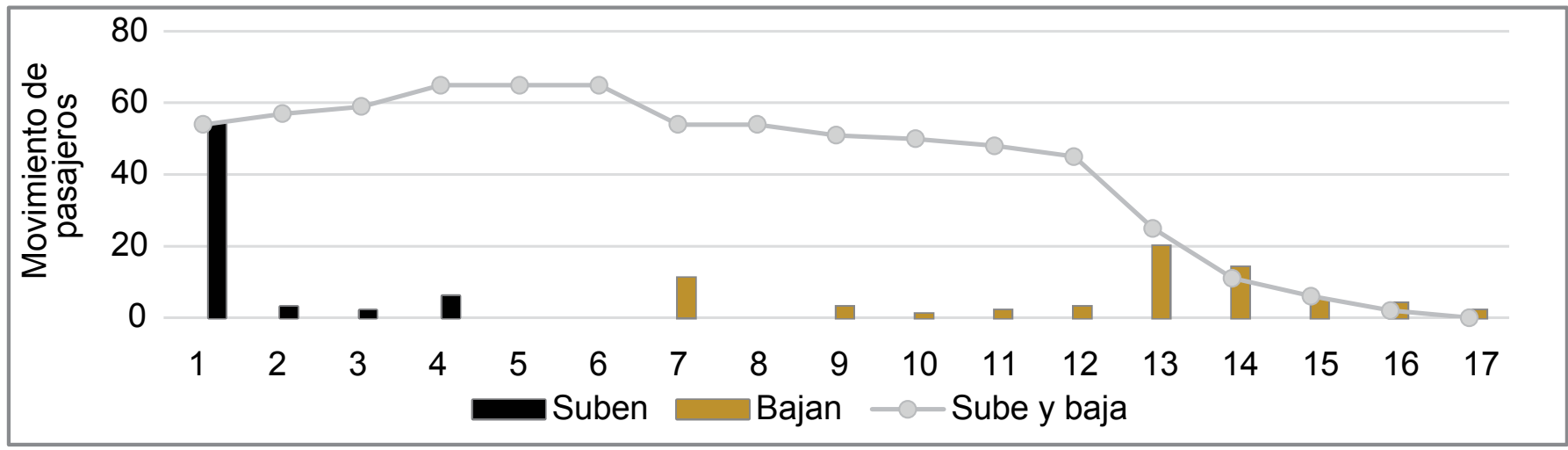

Figura 3. Sube y baja ruta UCR-Heredia

de viaje puntos en los cuales se pueden hacer modificaciones a las diferentes rutas. Por ejemplo, en un kilómetro de recorrido entre el centro de Guadalupe y el cruce de la Guaria, en Moravia, se detectó un tiempo de viaje de hasta 25 minutos, dado que en el bus de Heredia no suben ni bajan pasajeros entre el campus y Tibás se podrían evaluar recorridos alternativos que permitan el paso por sectores menos congestionados. Se pueden hacer estudios, por ejemplo, para reactivar el carril exclusivo para autobuses durante el período pico de la tarde, al costado norte del campus.

\section{Indicadores de desempeño}

De acuerdo a los indicadores de desempeño previamente mencionados, se aplicaron estos en las 17 rutas de servicio especial. Los resultados se aprecian en la Tabla 7. Debido a que el sistema consiste en el transporte de estudiantes de la
Universidad de Costa Rica, se debe acoplar a los horarios lectivos. Por ejemplo, respecto a la frecuencia por intervalos entre cada unidad, el nivel de servicio es deficiente (LOS E). Jiménez, Rojas y Salas (2015) indican que la frecuencia está relacionada con la calidad del servicio y la optimización de los costos de operación. Para ciertas rutas, por ejemplo, se recomienda proveer el servicio con un intervalo de 30 minutos entre las 5:30 pm y las 7:30 pm.

\section{Percepción de Calidad del Servicio}

A partir de la cantidad de estudiantes matriculados, se obtuvo un tamaño de muestra estadísticamente significativo $(n=380)$. Donde entre otras preguntas, se solicitó que calificarán de 1 a 5 (siendo 1 nada satisfecho y 5 muy satisfecho) algunos elementos del sistema actual los resultados se encuentran en la Figura 4. Los aspectos que peor calificación recibieron son aquellos que se encuentran relacionados con las paradas existentes. 
Tabla 7. Análisis de rutas universitarias

\begin{tabular}{|c|c|c|c|c|c|}
\hline Ruta & $\begin{array}{c}\text { Frecuencia por intervalos } \\
\text { entre cada unidad }\end{array}$ & $\begin{array}{c}\text { Frecuencia } \\
\text { (cantidad de viajes diarios } \\
\text { salida del destino) }\end{array}$ & $\begin{array}{c}\text { Frecuencia } \\
\text { (cantidad de viajes diarios } \\
\text { salida de UCR) }\end{array}$ & $\begin{array}{l}\text { Horas de servicio } \\
\text { hacia UCR }\end{array}$ & $\begin{array}{l}\text { Horas de servicio hacia } \\
\text { destino }\end{array}$ \\
\hline Acosta & $\mathrm{F}$ & $\mathrm{E}$ & $E$ & $E$ & $E$ \\
\hline Alajuela & $\mathrm{E}$ & B & B & $E$ & $\mathrm{E}$ \\
\hline Alajuelita & $E$ & C & B & C & E \\
\hline Calle Blancos & $E$ & B & B & $C$ & C \\
\hline Coronado & $\mathrm{E}$ & A & A & $\mathrm{E}$ & D \\
\hline Desamparados & $\mathrm{E}$ & $A$ & A & $E$ & C \\
\hline Grecia & $\mathrm{F}$ & $\mathrm{D}$ & D & $\mathrm{E}$ & $\mathrm{E}$ \\
\hline Heredia & $E$ & A & A & C & C \\
\hline Pavas & $\mathrm{E}$ & A & B & D & C \\
\hline Tibás & $E$ & A & A & C & D \\
\hline Santa Ana Escazú & $E$ & $\mathrm{D}$ & C & $\mathrm{E}$ & E \\
\hline $\begin{array}{l}\text { El Carmen de } \\
\text { Goicochea }\end{array}$ & $E$ & $\mathrm{D}$ & D & D & $\mathrm{D}$ \\
\hline
\end{tabular}

Fuente: Elaboración propia utilizando los criterios de TCRP (2003).

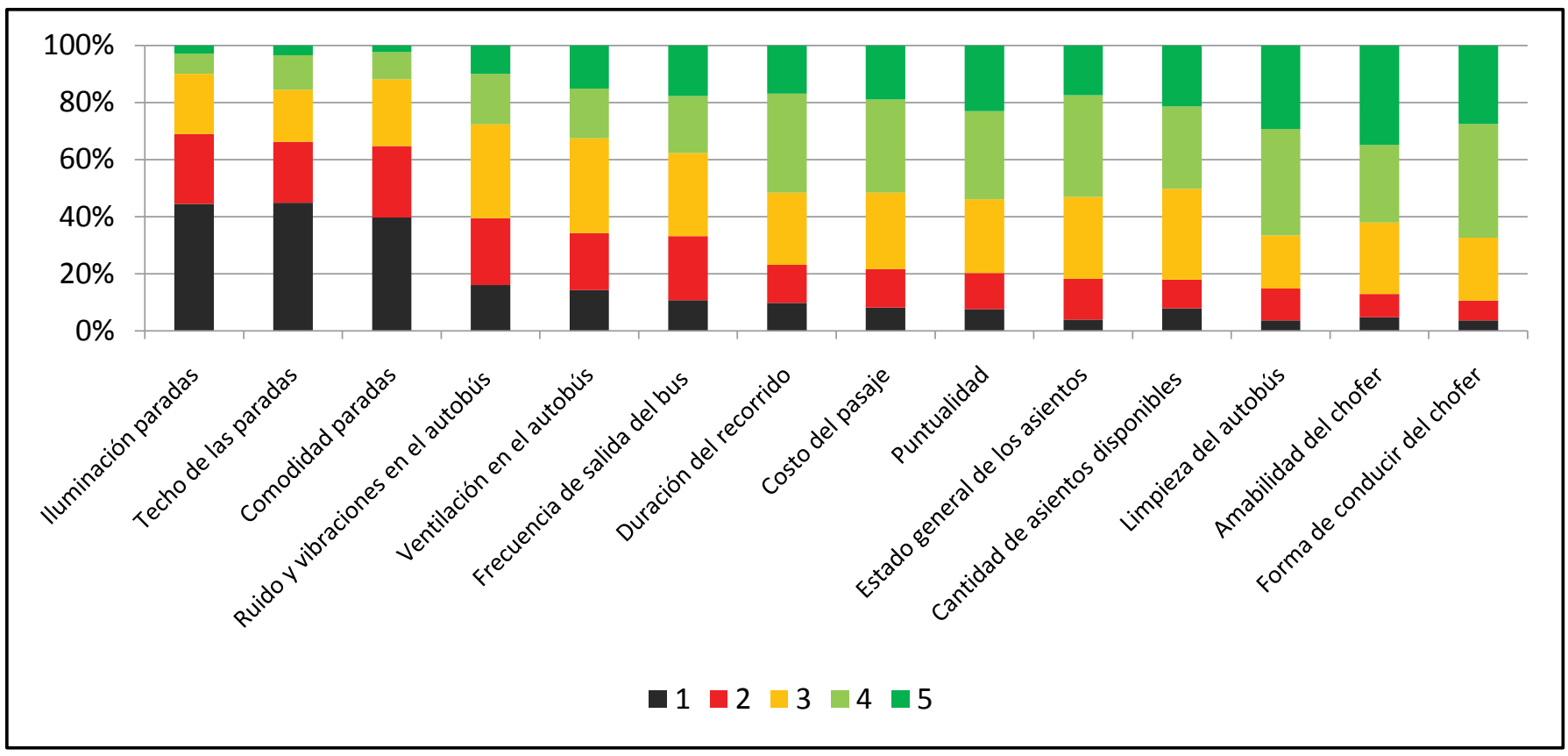

Figura 4. Calificación a los elementos que conforman el transporte público modalidad autobús. La percepción de la calidad varía del 1 al 5 , siendo 1 nada satisfecho y 5 muy satisfecho $(\mathrm{n}=380)$

Además, en la Figura 5 se detallan las acciones que el estudiantado considera más apropiadas para mejorar la calidad del servicio, las que resultaron más importantes para las personas usuarias corresponden a aspectos como mejorar la frecuencia de salida, mejorar la información disponible al usuario y la implementación de una terminal de buses. Por ejemplo, Jiménez, Rojas y Salas (2015) indican que una serie de estrategias pueden ser implementadas para atraer usuarios como la 


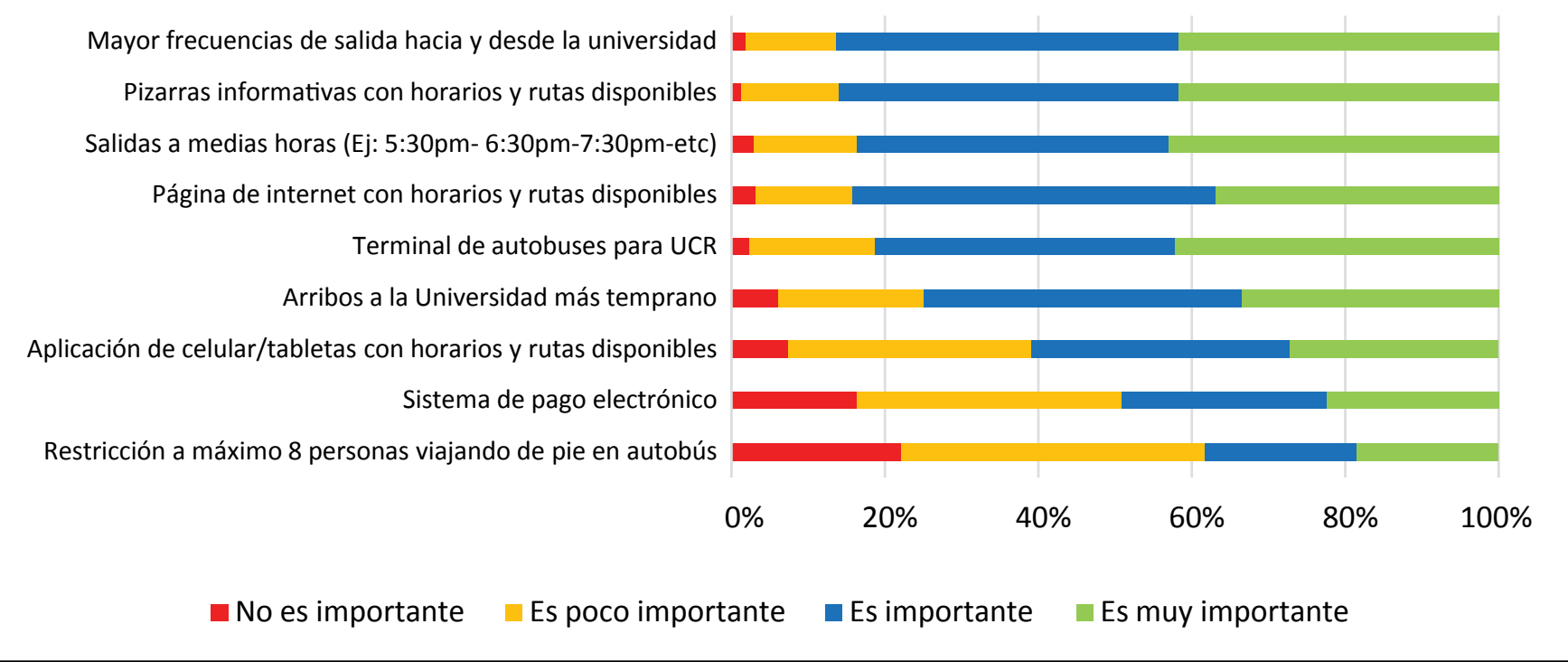

Figura 5. Prioridad de mejoras según el usuario $(n=380)$

construcción de paradas y estaciones terminales, aumento de la cobertura espacial, aumento de las frecuencias, mecanismos de información en tiempo real, mejoramiento de servicio al cliente, actualización del modelo tarifario, implementación de auditorías internas, entre otros.

\section{Cobertura de rutas}

Con respecto a la cobertura se realizó un levantamiento de acuerdo a las cantidades de estudiantes matriculados, y se ubicaron las 17 rutas que brindan servicio en la actualidad. La Figura 6 presenta un mapa con los distritos clasificados de acuerdo a la cantidad de estudiantes registrados y las rutas existentes de autobús UCR. Se determinó que existen distritos con potencial para la implementación de rutas nuevas, especialmente en lugares que no cuentan con rutas regulares de transporte público que pasen por las cercanías del campus.

Estos distritos corresponden a la Trinidad en Moravia, Ipís en Goicoechea, San Miguel en Desamparados, Tejar en El Guarco. También se determinó que una ruta entre el campus y San Isidro de El General de Pérez Zeledón tiene potencial, al menos para viajes de lunes en la mañana hacia el campus y viernes en la tarde hacia Perez Zeledón.

\section{CONCLUSIONES Y RECOMENDACIONES}

Se logra determinar el desempeño y la percepción de la calidad del servicio de transporte de estudiantes de la Sede Rodrigo Facio de la Universidad de Costa Rica. A partir de la evaluación del sistema se detectaron rutas con potencial de implementación. Tales como Trinidad de Moravia, San Miguel de Desamparados, Ipís de Goicoechea, Tejar de El Guarco y San Isidro de El General de Pérez Zeledón.

Es recomendable reducir los intervalos de salidas durante los periodos pico para las rutas de mayor demanda. Por otro lado, el promedio de calificación es 7,05 (en una escala del uno al diez) demuestra que la percepción de calidad cumple con un mínimo, pero con amplias posibilidades de mejora. A pesar de que la recopilación de información es relativamente simple, y de común utilización en otros países, en Costa Rica el material existente relacionado con indicadores de desempeño y calidad del servicio es escaso.

Según la percepción del usuario, los tres aspectos con menor calificación tienen dependencia con temas de infraestructura: iluminación, techo y comodidad global de las paradas. El transporte público requiere de la interacción de distintos elementos para que el servicio sea atractivo. Aceras, rampas, seguridad, condiciones de la calzada, ventilación, congestionamiento, disponibilidad de información, son sólo algunos aspectos evaluados. Finalmente, es imperativo que el país cuente con una política integral de transporte que priorice al transporte público, por encima del transporte privado, y que se implementen medidas que se han implementado en otros países para mejorar la movilidad urbana, tales como carriles exclusivos, sistemas de transporte masivo (tipo Bus Rapid Transit), entro otros. 


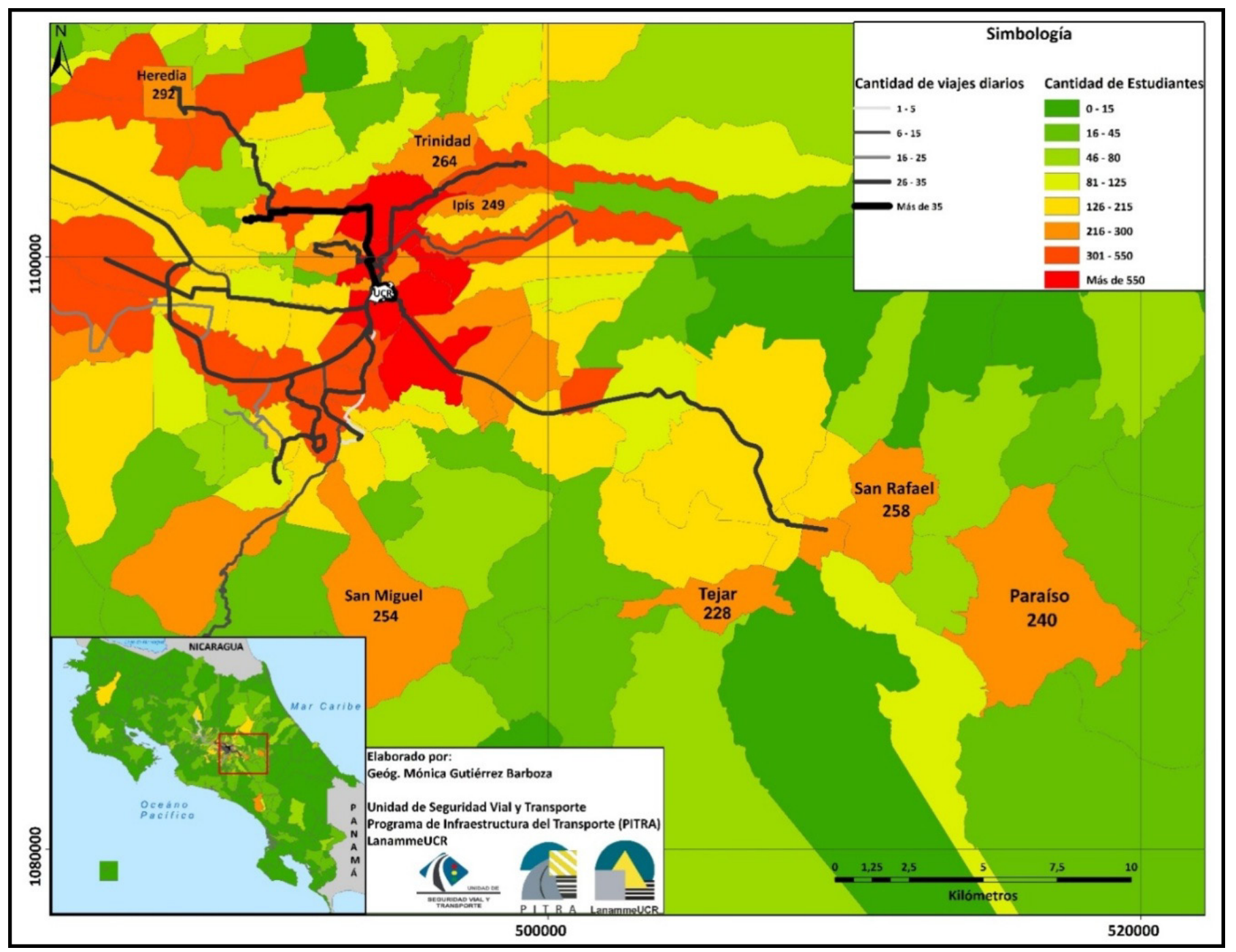

Figura 6. Mapa de la cantidad de viajes diarios reportados y de cantidad de estudiantes matriculados en la Sede Rodrigo Facio de la Universidad de Costa Rica.

\section{AGRADECIMIENTOS}

Los autores desean mostrar un agradecimiento a la geógrafa Mónica Gutiérrez Barboza, de la Unidad de Seguridad Vial y Transporte del Programa de Infraestructura del Transporte del LanammeUCR, por la elaboración del mapa presentado en este artículo. También se agradece a las personas que colaboraron, de manera voluntaria, con la administración de la encuesta de percepción de calidad del servicio. 


\section{REFERENCIAS BIBLIOGRÁFICAS}

1. Brenes, E. y Rodríguez, F. (2008). Propuesta de desarrollo urbano costarricense. Antecedentes, alternativas y desafíos. Revista Geográfica de América Central, $1(41), 77-119$.

2. Burgos, C., Silva, C., Troncoso, S. y Franco, B. (2013). Lo cotidiano en el transporte público de Culiacán: hacia una movilidad urbana sostenible y segura. URBS. Revista de Estudios Urbanos y Ciencias Sociales, 3(2), 123-139.

3. Contreras (1982) Análisis de datos y uso de modelos en rutas de autobuses. Aplicación en el sector Tibás. Informe de proyecto final para graduación. Universidad de Costa Rica

4. Eboli, L. y Mazzulla, G. (2011). A methodology for evaluating transit service quality based on subjective and objective measures from the passenger's point of view. Transport Policy, 18(1), 172-181.

5. Hernández, H. y Jiménez, D. (2014) Encuesta de Transporte 2013. Sede Rodrigo Facio. Universidad de Costa Rica. Programa Infraestructura del Transporte. Laboratorio Nacional de Materiales y Modelos Estructurales.

6. INECO. (2011). Plan Nacional de Transportes de Costa Rica (2011-2035) Transporte público: el transporte público de pasajeros y el proyecto de sectorización.

7. Iracheta, A. (2011). La necesidad de una política pública para el desarrollo de sistemas integrados de transporte en grandes ciudades mexicanas. Revista INVI, 26(71), 133-142.

8. Jiménez, J., Rojas, A. y Salas, M. (2015) Tariff Integration for Public Transportation in the Metropolitan Area of Bucaramanga. INGE CUC, 11(1), 25-33.

9. Leandro, M., Molina, O., y Riba, L. (2013). Intersecciones de la movilidad activa: construcción interdisciplinar del espacio público en el campus universitario. On the w@terfront, 28, 40-56.

10. Litman, T. (2003). Measuring transportation: traffic, mobility and accessibility. ITE journal, 73 (10), 28-32

11. Mohring, H. (1972). Optimization and scale economies in urban bus transportation. The American Economic Review, 62(4), 591-604.

12. Ortúzar, J. (2000). Modelos de demanda de transporte. Alfaomega; Universidad Católica de Chile.

13. Ortúzar, J. y Willumsen, L. (2011). Modelling Transport. (4th Edition). Wiley.

14. Otoya, M. (2013). Estimación económica de las principales deseconomías presentes en el Gran Área Metropolitana (GAM) de Costa Rica. Revista Iberoamericana de Economía Ecológica, 13, 15-27.

15. Pardo, C. (2009). Los cambios en los sistemas integrados de transporte masivo en las principales ciudades de América Latina.

16. Schipper, L., Saenger, C., \& Sudardshan, A. (2011). Transport and carbon emissions in the United States: the long view. Energies, 4(4), 563-581.

17. TCRP. (2002). Report 88. A guidebook for Developing a Transit Performance-Measurement System. Transit Cooperative Research Program. Transportation Research Board.

18. TCRP. (2003). Report 100. Transit Capacity and Quality of Service Manual. Second Edition. Transit Cooperative Research Program. Transportation Research Board.

19. TCRP. (2013). Report 165. Transit Capacity and Quality of Service Manual. Third Edition. Transit Cooperative Research Program. Transportation Research Board.

20. Van Reeven, P. (2008). Subsidisation of urban public transport and the Mohring effect. Journal of Transport Economics and Policy,42(2), 349-359. 